\title{
Die Anwendung von Kaliumferricyanid in alkalischer Lösung zur Bestimmung von Vanadium und Chrom.
}

\author{
Von \\ Howard E. Palmer. ${ }^{1}$
}

In früheren Mitteilungen aus diesem Laboratorium sind Methoden beschrieben worden für die Bestimmung von Cer neben anderen seltenen Erden, ${ }^{2}$ von Thallium ${ }^{3}$ und von Arsen, Antimon und Zinn, ${ }^{4}$ die basiert waren auf der Oxydationswirkung von Kaliumferricyanid in alkalischer Lösung und Oxydation des entstehenden Ferrocyanids durch Permanganat in saurer Lösung.

In der vorliegenden Mitteilung wird die Anwendung dieser Reaktionen auf die Bestimmung von Vanadium und Chrom beschrieben. Die folgenden Gleichungen stellen die Reaktionen dar:

$$
\begin{gathered}
\mathrm{V}_{2} \mathrm{O}_{4}+2 \mathrm{~K}_{3} \mathrm{Fe}(\mathrm{CN})_{6}+2 \mathrm{KOH}=\mathrm{V}_{2} \mathrm{O}_{5}+2 \mathrm{~K}_{4} \mathrm{Fe}(\mathrm{CN})_{6}+\mathrm{H}_{2} \mathrm{O} \\
\mathrm{Cr}_{2} \mathrm{O}_{3}+6 \mathrm{~K}_{3} \mathrm{Fe}(\mathrm{CN})_{6}+6 \mathrm{KOH}=2 \mathrm{CrO}_{3}+6 \mathrm{~K}_{4} \mathrm{Fe}(\mathrm{CN})_{6}+3 \mathrm{H}_{2} \mathrm{O} \\
5 \mathrm{~K}_{4} \mathrm{Fe}(\mathrm{CN})_{6 j}+\mathrm{KMnO} \mathrm{K}_{7}+4 \mathrm{H}_{2} \mathrm{SO}_{4}=5 \mathrm{~K}_{3} \mathrm{Fe}(\mathrm{CN})_{6}+3 \mathrm{~K}_{2} \mathrm{SO}_{4}+ \\
\mathrm{MnSO}_{4}+4 \mathrm{H}_{2} \mathrm{O} .
\end{gathered}
$$

\section{Bestimmung von Vanadium.}

Eine Lösung von Ammoniumvanadat wurde hergestellt durch Auflösen des reinen Salzes in Wasser; ihren Gehalt bestimmte man durch Eindampfen gemessener Mengen im Platintiegel und Wägung vom Vanadinpentoxyd nach dem Glühen.

Durch bestimmte Mengen dieser Lösung von Ammoniumvanadat wurde nach schwachem Ansäuern mit Salzsäure ein Strom von Schwefeldioxyd geleitet, bis die klare blaue Farbe vollständige Re-

1 Aus dem Amer. Journ. Science (Sill.) ins Deutsche übertragen von I. KoppeL-Berlin.

2 Z. anorg. Chem. 59, 71 .

3. anorg. Chem. 62, 218.

'Z. anorg. Chem. 67, 317 . 
duktion $z \mathrm{U} \mathrm{V}_{2} \mathrm{O}_{4}$ anzeigte. Die Lösung kochte man dann im Kohlendioxydstrom, bis die letzten Spuren von Schwefeldioxyd entfernt waren. Die abgekühlte Lösung versetzte man mit wenigstens $10 \mathrm{mal}$ so viel Ferricyanid, als theoretisch zur Oxydation erforderlich war, und mit einer Lösung von $6 \mathrm{~g}$ Kaliumhydroxyd. Die Lösungen von Ferricyanid und Kaliumhydroxyd müssen hinreichend konzentriert sein, so dafs das gesamte Volumen der Lösung nur 100 oder $125 \mathrm{ccm}$ beträgt. Es zeigte sich, dals wenigstens die angegebenen Mengen von Ferricyanid und Kaliumhydroxyd zur vollständigen Oxydation in der angegebenen Verdünnung erforderlich sind, und dafs bei stärkerer Verdünnung mehr Ferricyanid gebraucht wird. Es erwies sich als notwendig, das Vanadium vor dem Ansäuern und der Titration mit Permanganat zu entfernen, da es sonst mit dem Ferrocyanid einen Niederschlag bildet. Die Entfernung des Vanadiums erfolgte durch eine Lösung von Bariumhydroxyd, die es vollständig als Bariumvanadat ausfällt. Der Niederschlag wurde nach dem Absitzen über Asbest abgesaugt, Filtrat und Waschwässer mit Salzsäure angesäuert und mit Permanganat titriert. Titration in schwefelsaurer Lösung gab keine zufriedenstellenden Resultate, weil es schwierig war, den Endpunkt in Gegenwart des gebildeten Bariumsulfats zu erkennen; auch bei früheren Untersuchungen hatte sich schon gezeigt, dafs anders als bei Ferrosalzen, das Ferrocyanid in der Kälte in Gegenwart verdünnter Salzsäure mit Permanganat titriert werden kann. Die Resultate der Bestimmungen sind in Tabelle 1 zusammengestellt.

Tabelle 1.

\begin{tabular}{c|c|c|c|c|c}
\hline \hline $\mathrm{Nr}$. & $\begin{array}{c}\text { Angew. } \\
\mathrm{V}_{2} \mathrm{O}_{5} \\
\mathrm{~g}\end{array}$ & $\begin{array}{c}\text { Angew. } \\
\mathrm{K}_{3} \mathrm{Fe}(\mathrm{CN})_{6} \\
\mathrm{~g}\end{array}$ & $\begin{array}{c}\text { Angew. } \\
\mathrm{KOH} \\
\mathrm{g}\end{array}$ & $\begin{array}{c}\text { Gefunden } \\
\mathrm{V}_{2} \mathrm{O}_{5} \\
\mathrm{~g}\end{array}$ & $\begin{array}{c}\text { Fehler } \\
\mathrm{g}\end{array}$ \\
\hline \hline 1 & 0.0960 & 4 & 6 & 0.0959 & -0.0001 \\
2 & 0.0960 & 4 & 6 & 0.0954 & -0.0006 \\
3 & 0.0960 & 4 & 6 & 0.0956 & -0.0004 \\
4 & 0.0960 & 4 & 6 & 0.0962 & +0.0002 \\
5 & 0.0960 & 4 & 6 & 0.0956 & -0.0004 \\
6 & 0.0960 & 4 & 6 & 0.0959 & -0.0001 \\
7 & 0.0960 & 4 & 6 & 0.0961 & +0.0001 \\
8 & 0.0960 & 4 & 6 & 0.0961 & +0.0001 \\
9 & 0.0960 & 4 & 6 & 0.0960 & \pm 0.0000 \\
10 & 0.0960 & 4 & 6 & 0.0961 & +0.0001 \\
\end{tabular}

Z. anorg. Chem, Bd. 67. 


\section{Bestimmungen von Chrom.}

Wie Bollenbach und LuchmanN ${ }^{1}$ neuerdings gezeigt haben, kann Chrom quantitativ von $\mathrm{Cr}_{2} \mathrm{O}_{3}$ zu $\mathrm{CrO}_{3}$ durch Kaliumferricyanid in alkalischer Lösung oxydiert werden, und man erhält ein Mals für die Grölse der Oxydation durch Titration des gebildeten Ferrocyanids mit Permanganat. Nach ihrer Methode wurde ein Überschufs von wenigstens der 4-6 fachen theoretischen Menge an Kaliumferricyanid und $40-50 \mathrm{ccm}$ einer 2-norm. Natriumhydroxydlösung zur Chromlösung hinzugefügt, um vollständige Oxydation des Chroms zu bewirken. Das oxydierte Chrom wurde durch Fällung als Bariumchromat mit Bariumhydroxyd und Filtration entfernt. Das Filtrat säuerte man mit Chlorwasserstoff an und titrierte mit Permanganat nach Bollenbachs Abänderung von De Haen Methode, ${ }^{2}$ wodurch die Schwierigkeit überwunden werden soll, die darin besteht, dals man bei der Titration grofser Nlengen von Ferrocyanid den Endpunkt schlecht erkennt wegen der Bildung eines Niederschlages von $\mathrm{K}_{2} \mathrm{MnFe}(\mathrm{CN})_{6}$ während der Titration, wie zuerst GRÜTZNER $^{3}$ gezeigt hat. Diese Abänderung bestand darin, dals man zuerst einen Überschuls von Permanganat zur Lösung hinzufügte, und nachdem der Niederschlag verschwunden war, den Überschufs von Permanganat mit $1 / 20$-norm. Kaliumferrocyanid in Gegenwart einer Spur Ferrisalz zurücktitrierte, wobei die Bildung einer dauernden Grünfärbung von Ferriforrocyanid den Endpunkt anzeigte.

Bei Versuchen zur Prüfung dieses Verfahrens kam eine Lösung von Kaliumchromat zur Anwendung, deren Gehalt bestimmt war durch Fällen bestimmter Mengen als Merkurochromat und Wägen als Chromioxyd nach dem Glühen; ferner wurde der Gehalt auch bestimmt durch Fällung von Chromihydroxyd durch Ammoniak nach Reduktion mit Salzsäure und Alkohol.

Die Bestimmungen wurden folgendermalsen ausgeführt: Gemessene Mengen der Lösung von bekanntem Gehalt wurden schwach salzsaner gemacht und mit Schwefeldioxyd behandelt, bis die klare Grünfärbung vollständige Reduktion anzeigte. Das Schwefeldioxyd vertrieb man durch Kochen im Kohlendioxydstrom. Die abgekühlte Lösung versetzte man mit Kaliumferricyanid und Kaliumhydroxyd

1 Z. anorg. Chem. 60, 446.

"Zeitschr. analyt. Chem. 47, 687.

${ }^{3}$ Chem. Centrbl, 1902 I, 500. 
in Lösung nach dem oben beschriebenen Verfahren von BolLEnBaCH und LuChMaNN.

Als man jedoch die Reagenzienmengen benutzte, die die genannten Autoren als ausreichend für die Oxydation des Chroms bezeichnet haben, erhielt man die niedrigen Resultate von Tabelle 2, woraus hervorgeht, dals die Oxydation unvollständig war. Als man dagegen das 15 fache der theoretischen Menge von Kaliumferricyanid und eine ziemlich starke Lösung von Kaliumbydroxyd bei einem Gesamtvolumen von 100-125 ccm anwandte, wie bei den Versuchen der Tabelle 3, erhielt man Resultate, die mit den theoretischen übereinstimmten.

Tabelle 2.

\begin{tabular}{c|c|c|c|c|c}
\hline \hline $\mathrm{Nr}$. & $\begin{array}{c}\text { Angew. } \\
\mathrm{CrO}_{3} \\
\mathrm{~g}\end{array}$ & $\begin{array}{c}\text { Angew. } \\
\mathrm{K}_{\mathbf{3}} \mathrm{Fe}(\mathrm{CN})_{6} \\
\mathrm{~g}\end{array}$ & $\begin{array}{c}\text { Angew. } \\
\mathrm{KOH} \\
\mathrm{g}\end{array}$ & $\begin{array}{c}\text { Gefunden } \\
\mathrm{CrO}_{3} \\
\mathrm{~g}\end{array}$ & $\begin{array}{c}\text { Fehler } \\
\mathrm{g}\end{array}$ \\
\hline & 0.1010 & 6 & 12 & 0.0979 & -0.0031 \\
1 & 0.1010 & 8 & 16 & 0.0981 & -0.0029 \\
3 & 0.1010 & 8 & 16 & 0.0989 & -0.0021 \\
4 & 0.1010 & 8 & 12 & 0.0997 & -0.0013
\end{tabular}

Tabelle 3 .

\begin{tabular}{c|c|c|c|c|c}
\hline $\mathrm{Nr}$. & $\begin{array}{c}\text { Angew. } \\
\mathrm{CrO}_{3} \\
\mathrm{~g}\end{array}$ & $\begin{array}{c}\text { Angew. } \\
\mathrm{K}_{3} \mathrm{Fe}(\mathrm{CN})_{6} \\
\mathrm{~g}\end{array}$ & $\begin{array}{c}\text { Angew. } \\
\mathrm{KOH} \\
\mathrm{g}\end{array}$ & $\begin{array}{c}\text { Gefunden } \\
\mathrm{CrO}_{3} \\
\mathrm{~g}\end{array}$ & $\begin{array}{c}\text { Fehler } \\
\mathrm{g}\end{array}$ \\
\hline \hline 1 & 0.1010 & 16 & 15 & 0.1016 & +0.0006 \\
2 & 0.1010 & 16 & 15 & 0.1014 & +0.0004 \\
3 & 0.1010 & 16 & 15 & 0.1013 & +0.0003 \\
4 & 0.0505 & 16 & 15 & 0.1514 & +0.0009 \\
5 & 0.0505 & 16 & 15 & 0.0506 & +0.0001
\end{tabular}

Wie bei den früheren Untersuchungen mufste eine Korrektur angebracht werden wegen der Permanganatmenge, die das Ferricyanid allein verbraucht.

\section{Bestimmung von Vanadium und Chrom nebeneinander.}

Für die Bestimmung von Vanadium und Chrom nebeneinander wurde das folgende Verfahren ausgearbeitet. Man teilte die Lösung, die beide Elemente in der höheren Oxydationsstufe enthielt, in zwei Teile. Den einen Teil der Lösung behandelte man nach schwachem 
Ansäuern mit Salzsäure mit Schwefeldioxyd, bis zur vollständigen Reduktion von Vanadium und Chrom. Dann kochte man die Lösung im Kohlendioxydstrom, bis die letzten Spuren von Schwefeldioxyd ausgetrieben waren. Die kalte Lösung versetzte man mit genügenden Mengen Kaliumferricyanid und Kaliumbydroxyd in gelöster Form, wobei das Chromisalz in $\mathrm{CrO}_{3}$, das Vanadium aus dem Tetroxyd in Pentoxyd überging. Nachdem die Flüssigkeit einige Minuten gestanden hatte, wurde eine Lösung von Bariumbydroxyd bis zur vollständigen Fällung hinzugesetzt. Der Niederschlag ron Barium. chromat und Bariumvanadat wurde über Asbest abfiltriert und sorgfältig gewaschen. Das Filtrat säuerte man mit verdünnter Chlor. wasserstoffsäure an und versetzte mit einer bekannten Menge Permanganat im Überschufs, worauf dieser Überschufs mit $1 / 21^{-}$ norm. Kaliumferrocyanid nach dem oben angegebenen Verfahren zurücktitriert wurde.

In dem andern Teil der Lösung wurde Vanadium folgendermafsen bestimmt. Die Lösung, deren Volumen etwa $100 \mathrm{ccm}$ betrug, versetzte man mit 10-15 ccm Eisessig und mit Wasserstoffsuperoxyd, sodann erhitzte man sie mehrere Minuten zum Sieden; hierdurch wurden Perchromsäure und Pervanadinsäure, die sich zuerst in der Kälte gebildet haben, zerstört, wobei das Chrom in die dreiwertige Form überging, während das Vanadium in fünfwertiger Form verblieb. Man verdünnte die Lösung etwas und setzte eine Lösung von Bleiacetat hinzu, um Bleivanadat vollständig auszufällen; das Chrom fiel natürlich nicht aus. Die Lösung wurde heftig gerührt, um den Niederschlag zusammenzuballen und sodann zum Sieden erhitzt, wodurch der Niederschlag kompakter wurde. Man filtrierte ihn dann über Asbest ab, wusch sorgfältig und löste in Kaliumhydroxyd. Die Lösung wurde stark mit Schwefelsäure angesäuert, wobei das Blei als Sulfat ausfiel, während die Vanadinsäure in Lösung blieb. Ein Strom von Schwefeldioxyd wurde dann bis zur vollständigen Reduktion des Vanadiums durch die Lösung geleitet: das Schwefeldioxyd vertrieb man durch Kochen im Kohlensäurestrom und die warme Lösung titrierte man mit Permanganat bis zur Rotfärbung, die man leicht auch in Gegenwart des weifsen Bleisulfats erkennen konnte.

Diese Titration ergab die Menge des vorhandenen Vanadiums und durch Subtraktion der hierfür verbrauchten Permanganatmenge von der bei der ersten Titration verbrauchten Menge erhielt man die Anzahl Kubikzentimeter Permanganat, die für die Oxydation 
von Chromioxyd zu Chromtrioxyd verbraucht sind. Die Resultate der Bestimmungen sind in Tabelle 4 zusammengestellt.

Tabelle 4.

\begin{tabular}{r|c|c|c|c|c|c}
\hline $\mathrm{Nr}$. & $\begin{array}{c}\text { Angew. } \\
\mathrm{V}_{2} \mathrm{O}_{5} \\
\mathrm{~g}\end{array}$ & $\begin{array}{c}\text { Angew. } \\
\mathrm{CrO}_{3} \\
\mathrm{~g}\end{array}$ & $\begin{array}{c}\text { Gefund. } \\
\mathrm{V}_{2} \mathrm{O}_{5} \\
\mathrm{~g}\end{array}$ & $\begin{array}{c}\text { Fehler } \\
\mathrm{g}\end{array}$ & $\begin{array}{c}\text { Gefund. } \\
\mathrm{CrO}_{3} \\
\mathrm{~g}\end{array}$ & $\begin{array}{c}\text { Fehler } \\
\mathrm{g}\end{array}$ \\
\hline \hline 1 & 0.1139 & 0.1010 & 0.1134 & -0.0005 & 0.1010 & \pm 0.0000 \\
2 & 0.1139 & 0.1010 & 0.1139 & \pm 0.0000 & 0.1017 & +0.0007 \\
3 & 0.1139 & 0.1010 & 0.1134 & -0.0000 & 0.1019 & +0.0009 \\
4 & 0.1139 & 0.1010 & 0.1142 & +0.0003 & 0.1019 & +0.0009 \\
5 & 0.1139 & 0.1010 & 0.1131 & -0.0008 & 0.1015 & +0.0005 \\
6 & 0.1139 & 0.1010 & 0.1134 & -0.0005 & 0.1016 & +0.0006 \\
7 & 0.1139 & 0.0505 & 0.1139 & \pm 0.0000 & 0.0507 & +0.0002 \\
8 & 0.1139 & 0.0505 & 0.1134 & -0.0005 & 0.0507 & +0.0002 \\
9 & 0.0569 & 0.0505 & 0.0565 & -0.0004 & 0.0505 & \pm 0.0000 \\
10 & 0.0569 & 0.0505 & 0.0563 & -0.0006 & 0.0508 & +0.0003 \\
& & & & & &
\end{tabular}

New Haven, U.S. A., The Kent Chemical Laboratory of Yale University.

Bei der Redaktion eingegangen am 22. Juni 1910. 